\title{
The Fruits of Fiber: The Invention of the Flexible Fiberoptic Gastroscope
}

\author{
Jonathan D. Kaunitz
}

Published online: 6 September 2014

(C) Springer Science+Business Media New York (Outside the USA) 2014

May it be a light to you in dark places, when all other lights go out.

- J.R.R. Tolkien, The Fellowship of the Ring

There is a single light of science, and to brighten it anywhere is to brighten it everywhere.

— Isaac Asimov

...for to predict the future accurately is to invent, and to invent is to see something that is not yet here, that is the paradox

—Basil I. Hirschowitz

One often hears about the humble beginnings of some of the largest industries, with early prototypes of personal computers put together in a garage by a group of students, spawning a culture-changing, multibillion dollar global industry. In our field of gastroenterology, we increasingly view research as involving multisite consortia, 8-figure budgets, million dollar instrumentation, and teams of bioinformatics experts and statisticians poring over terabytes of data. The garage or dorm room might have been a great place to start Google, Disney, Facebook, or Amazon, but are certainly not places for serious medical research. Yet, as you read on, this was the setting in which the fiberoptic endoscope, arguably the single greatest diagnostic tool for our profession in the last century, was invented.

In this fifth installment of "Paradigm Shifts in Perspective," Mel Wilcox and I would like to spotlight an invention which singlehandedly transformed gastroenterology into the field that it is-the flexible endoscope. It is

J. D. Kaunitz $(\square)$

Departments of Medicine and Surgery, West Los Angeles VA Medical Center, UCLA School of Medicine, 11301 Wilshire Blvd, Bldg 114, Room 217E, Los Angeles, CA 90073, USA e-mail: jake@ucla.edu difficult to believe that prior to 1960 , endoscopy was limited mostly to rigid sigmoidoscopy. On rare occasions, gastroscopy was conducted with a multi-hinged tube that required enormous skill combined with extreme cooperation and discomfort tolerance from its subjects, since one needed to align the oral cavity with the pharynx and esophagus through positioning and contortions that only a sword swallower would appreciate. Yet, many practitioners were adept at this, notably Rudolf Schindler, whose early works grace the first issues of this journal.

The history of gastroscopy is relatively recent, extending back only to the nineteenth century $[1,2]$. Although many had developed instruments equipped with optics that could be inserted into the body enabling the visualization of structures, it was not until 1881 that Johannes von Mickulicz developed the first working gastroscope, complete with air insufflation and integral light source. Following that advance, many inventors, engineers, and physicians jumped in, each with their own version and modification. The next advance was the semi-flexible endoscope in which one part consisted of multiple hinged segments with a series of lenses or prisms to conduct the light and wires to straighten the tube once inserted. Kelling first introduced this type in 1897 , followed by versions designed by Kuttner and Sussman. The advantages to these instruments were that the need for neck hyperextension for insertion was reduced, as was gastric intubation. The wires guiding the instrument's curvature were controlled by a wheel near the eyepiece, which is basically the same design as is used today to manipulate the endoscope head. It was Rudolf Schindler, however, who standardized and systematized foregut endoscopy, publishing his findings in early editions of the forerunner of this journal, The American Journal of Digestive Diseases and Nutrition, subject to a recent commentary in our January issue [3, 4]. It was 


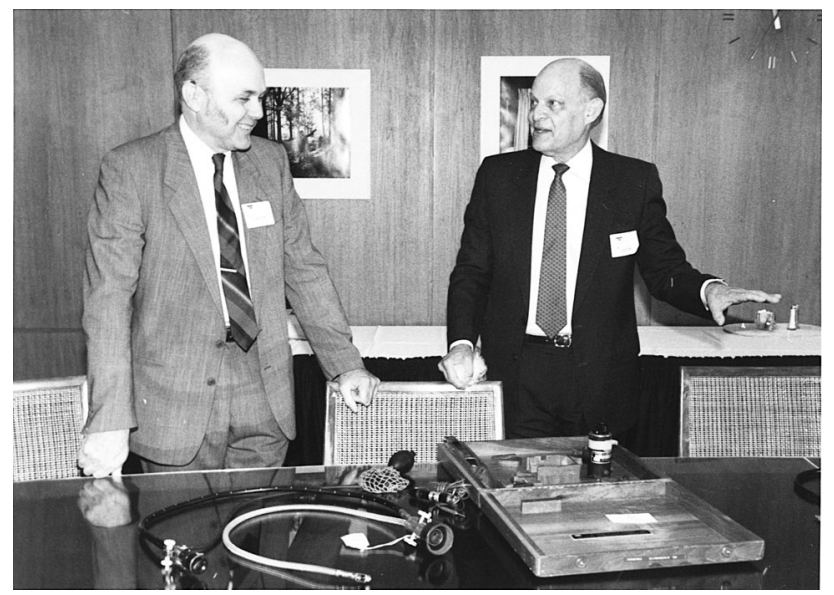

Fig. 1 Drs. Curtis and Hirschowitz at the Smithsonian Institute in 1988 with their prototype fiberoptic endoscope. (Photo courtesy Dr. Basil Hirschowitz)

Schindler who founded the American Gastroscopic Club, precursor of the American Society for Gastrointestinal Endoscopy, and trained hundreds of fellows in his art.

The next major advance in endoscopy is the focus of this Perspective, namely the invention of the flexible fiberoptic endoscope by Basil Hirschowitz and Larry Curtiss [5], who remarkably produced the first practical prototype of a flexible fiberoptic gastroscope in the late 1950s. In his published memoir, Dr. Hirschowitz [6] describes in detail his painstaking attention to detail combined with persistence despite considerable resistance from the naysayers, detractors, "experts," and even his senior advisor. Although the concepts of total internal reflection, image transmission through light fibers, and the concept of a flexible gastroscope predated Dr. Hirschowitz' invention by over 100 years, it was the creativity and ingenuity of Dr. Hirschowitz' group that facilitated the production of a practical working endoscope.

Perhaps the greatest single advance made during process of invention of the flexible endoscope was the fabrication of glass-clad optical glass fibers. Although probably unknown except to a tiny percentage of gastroenterologists, light guides prior to this had been hampered by marked signal loss, low resolution, and coloration from the use of low-grade glass combined with unsuccessful attempts to coat each fiber with oils, metals, plastics, and lacquers in order to increase internal reflectivity [7]. It was known for over a century that light is totally internally reflected at the interface of materials of high and low refractivity at angles nearly parallel to the interface, and since 1951, that cladding of glass fiber with a material of a lower refractive index would decrease reflective loss [7]. Nevertheless, it was not until Larry Curtiss, an undergraduate working in a

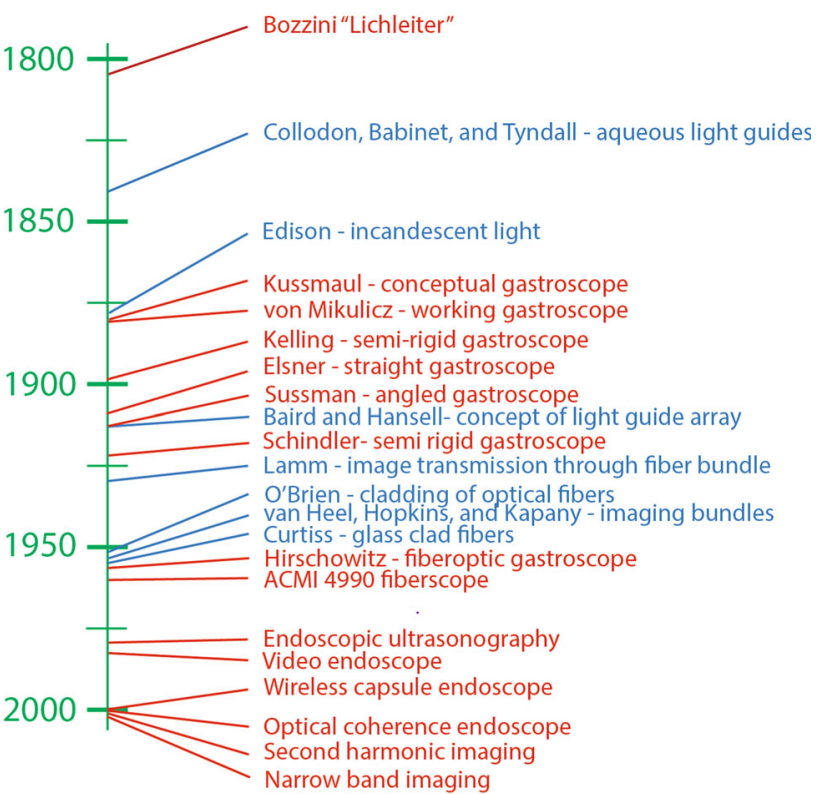

Fig. 2 Timeline depicting some of the major advances in instrumentation (red) and the scientific breakthroughs that underlie the technical advances (blue) leading to, and resulting from the development of the fiberscope

basement physics laboratory at the University of Michigan with Dr. Hirschowitz, then a GI fellow, inserted, against the advice of his advisors and "experts," a rod of optical glass into a tube of low-refractive glass, melted them together in a furnace, drew them through a tiny hole, and famously wound them on a container of "Mother's Oats" $[2,6,7]$. In this manner, the modern optical fiber was born. It was a matter of assembling and orienting these fibers and polishing their ends that enabled the production of the first modern high-resolution fiberoptic endoscope (Fig. 1). With this advance, the fiberoptic gastroscope and every other conceivable flexible optical transmission device could be manufactured. Dr. Wilcox will begin his tale where this one ends, in 1960, with the introduction of the first commercial fiberoptic endoscope, the American Cystoscopy Makers Model 4990.

The fiberoptic endoscope revolutionized the practice of gastroenterology, as Dr. Wilcox will describe, enabling its practitioners to easily visualize areas that had previously only been visible to the surgeon or to the pathologist, and opening the field of diagnostic and therapeutic endoscopy. Although considered one of the most influential advances to medicine in the second half of the twentieth century, the overall significance of fiberoptics is so great that its contributions to medicine are a mere historical footnote. After Curtiss demonstrated the superiority of glass-clad fibers, many improvements followed, notably the use of fused silica for the core material developed by Charles Kuen Kao, who won the Nobel Prize in for his visionary 
development of fiberoptics for long-distance data transmission [7]. Although numerous improvements in the design and manufacture of fiberoptic cables have occurred since 1957 (Fig. 2; [8, 9]), the glass-clad fiber invented by the then teenaged Curtiss remains the industry standard for low-loss light transmission. Let us hope that junior investigators now and in the future will continue to show the creativity, persistence, foresight, and vision of Drs. Curtiss and Hirschowitz as they pioneer new breakthroughs that will further shift the paradigm.

\section{References}

1. Hirschowitz BI. Development and application of endoscopy. Gastroenterology. 1993;104:337-342.
2. Vilardell F. Digestive Endoscopy in the Second Millenium: From the Lichtleiter to Echoendoscopy. Madrid: Grupo Aula Médica, S.L.; 2006.

3. Schindler R. Gastroscopy with a flexible gastroscope. Am J Dig Dis Nutr. 1935;2:656-663.

4. Leung WD, Gelrud A. Ushering in a new era in gastroenterology: the flexible gastroscope. Dig Dis Sci. 2014;59:12-15.

5. Hirschowitz BI, Peters CW, Curtiss LE. Preliminary report on a long fiberscope for examination of stomach and duodenum. Med Bull. 1957;23:178-180.

6. Hirschowitz BI. A personal history of the fiberscope. Gastroenterology. 1979;76:864-869.

7. Hecht J. City of Light: The Story of Fiber Optics. New York: Oxford University Press; 1999.

8. Sivak MV. Gastrointestinal endoscopy: past and future. Gut. 2006;55:1061-1064.

9. Kwan V. Advances in gastrointestinal endoscopy. Intern Med J. 2012;42:116-126. 